\title{
Mesenchymal Stromal Cells Used for Cellular Cardiomyoplasty to Treat Myocardial Infarction and Heart Failure
}

\author{
Amarachukwu Okpala ${ }^{1}$ and Leya Joykutty ${ }^{1 \#}$ \\ ${ }^{1}$ American Heritage High School Plantation, Plantation, Florida, USA \\ \#Advisor: Leya Joykutty
}

\section{ABSTRACT}

Heart disease is one of the leading causes of morbidity and mortality worldwide. Two of these diseases are heart failure and myocardial infarction. In America alone, there are about 6.2 million people with heart failure [5], and every 40 seconds, a patient with a heart attack is recorded [5]. Myocardial infarction, known as a heart attack, occurs after the blocking or occlusion of a coronary artery, disabling the delivery of oxygenated blood to regions of the heart [16]. Heart failure, usually occurring after ischemic diseases like myocardial infarction, is where the heart loses the ability to pump a sufficient blood supply to meet the body's needs [3]. The major ways of treating heart failure and myocardial infarction today are either too expensive or hard to come by, so a new sort of treatment is direly needed. Cellular cardiomyoplasty, a form of cell therapy, is being looked into as a new way to treat these two and other cardiomyopathies. Additionally, though there have been a few cells that have shown a possibility of use for cardiomyoplasty, this review focuses on mesenchymal stem cells, specifically called mesenchymal stromal cells. The purpose of this review is to look into what cellular cardiomyoplasty is, how it may be used in the future, and how mesenchymal stromal cells have shown potential to be used for it.

\section{Introduction}

Cardiovascular diseases harm the function of the heart in both adults and children. Heart disease has been the leading cause of death for years, and can include diseases from coronary artery disease, arrhythmias (irregular heart rhythm), and heart defects present at birth (congenital heart defects) [12]. Two of the major cardiomyopathies include myocardial infarction and heart failure.

A myocardial infarction occurs when there is a sudden blockage of the heart's coronary arteries, or there is extremely slow blood flow $[8,16]$. The typical cause of blockages in the coronary arteries are formations of blood clots, or the buildup of plaque in the artery (known as atherosclerosis). In some cases it can be very minor and even go unnoticed, though that usually happens when a patient is going through a long, chronic disease [16]. In other cases, myocardial infarction can be the reason of death or the cause of a life-long disability, requiring the need of assist devices for the beating of the heart. Myocardial infarction can cause poor after-effects to the heart because of the heart's response to it concerning T cells [36]. T cells are part of the immune system. Though it has shown to be useful in mice models because of its ability to promote reparative fibrosis in a postmitotic organ, after a period of time, these cells can cause chronic damage to the heart, leading to chronic heart failure because of its stimulation of fibroids [27, 36].

Myocardial infarction often leads to heart failure due to the fact that after the blockage of a coronary artery, the heart may no longer pump to its full ability [35, 36, 37]. If the heart is not pumping enough blood, then oxygen isn't getting to the heart at the necessary rate. This creates a hypoxic environment, leading to cellular death or necrosis, and scar tissue formation. Though the heart can be sustained for a certain period of time, the formation of scar tissues 
in the heart will stop the heart from being able to pump blood efficiently to other parts of the body. When vital organs stop receiving the oxygen from blood that is necessary for survival, they start to shut down, eventually leading to death of the entire organism. As time has advanced, more and more techniques for longer life have been developed. Myocardial infarction, for one, doesn't kill as many people today as it did perhaps, 50 years ago. However, because of the increase of survivors from an initial heart attack, more and more people are being diagnosed with and are dying from heart failure $[35,37]$. Because of this increase, effective and available treatment for heart failure is in high demand.

Heart failure, as stated above, occurs when the heart no longer has the ability to pump blood to the rest of the body. Diseases that damage and weaken the heart include ischemic heart disease, high blood pressure, and diabetes, and are some of the major causes of heart failure [10]. Therapeutics today for heart failure today include left ventricular assist devices (LVAD), heart transplantations, and different kinds of drugs and medications.

Left ventricular assist devices (LVAD) are mechanical pumps that are surgically implanted into the left ventricle. Though they usually serve as a bridge for heart transplantation, they can also be used as a treatment for heart failure. These devices mechanically pump blood throughout the body with a heart that cannot sufficiently do this on its own [3]. LVAD's have grown in use over the decades, and have improved in terms of size, noise emission, and durability [10]. Though LVAD has been seen as helpful in many cases and has increased in use of treatment for heart failure, it is highly expensive, exceeding a cost of \$200,000 (234,808 in 2011) [3]. It also can cause some other complications which include bleeding, right ventricular failure, thromboembolism, primary device failure, and infection.

Heart transplantation, on the other hand, is the most effective treatment for heart failure today. The problem is, because the heart is a necessary organ in all humans, there is a major scarcity of heart donors, making this treatment very hard to come by. In the years $1987-2012$, there were 40,253 people on the waiting list to get a heart transplant. However, only about $67 \%$ of these people actually received a transplant. Though patients with heart transplants have mostly shown major recoverys, heart failure is still a growing and leading cause of death. This makes the need to find a new therapeutic way to treat heart failure increasingly dire, one that can keep up with the increasing number of patients.

As heart failure continues to advance in a patient, they may need to start taking medicines for treatment. Because there are certain drugs that specifically target a certain area in helping the failing heart, patients usually have to take an array of medicines. Pharmacological classes of treatment for heart failure include channel blockers, which reduce the heart rate, diuretics, which reduce excess fluid in the body and helps with the heart workload, and anticoagulants, or blood thinners [10]. These treatments have been seen to prolong life and improve heart function, however, none of them have shown the ability to completely reverse the effect of heart failure on a patient.

It is important to realize that the above treatments currently used for heart failure have saved many lives, even with their stated issues. The main problem is that heart failure remains one of the highest reasons of death worldwide, giving the need for a new technique/treatment for ischemic cardiomyopathies to lower the deaths by significant numbers.

Cellular cardiomyoplasty is being looked into as a treatment for myocardial infarction and heart failure, and to work against the effects of other diseases that harm the heart. This form of cell therapy uses tissue engineering to transplant cells from one tissue type into another tissue type [2]. There are a few cells that have been looked into in a clinical setting and have shown the potential to be used in cellular cardiomyoplasty. These cells include bone-marrow derived stem cells, embryonic stem cells, amniotic stem cells, and mesenchymal stromal cells [3, 5]. The goal of cellular cardiomyoplasty in terms of heart failure is to replace the damaged necrotic cardiomyocytes with functional myocytes from other tissue types, and to enhance the vascularization in ischemic myocardium (blocked arteries in the heart) [5]. The goal of this treatment in terms of myocardial infarction is to completely prevent heart failure from occurring by saving the myocardium from getting weaker, and regenerating heart-muscle cells [5].

The beginning of clinical research for cell therapy was started because of the rates of death from tissueweakening diseases in our global community. One stem cell, as stated above, that can be used in cell therapy is called embryonic stem cells. Though embryonic stem cells do have a possibility of catalysing cardiac regeneration, the legal and ethical issues present makes the use of these cells unlikely in the near future [5]. 
Because embryonic stem cells are not the only stem cells with the potential of stimulating cardiac regeneration, cellular cardiomyoplasty still may become a revolutionary treatment for heart failure and myocardial infarction. However, cell therapy is still being looked into, and all of the possible problems or complications that may be caused by it are not yet known. There have been some clinical trials where cells like mesenchymal stromal cells have improved the function of the infarcted heart of animals like rats or mice, however cellular cardiomyoplasty still needs to be tested in different stages to prove that it is safe and effective for use to treat myocardial infarction and heart failure. What is known, as shown in Figure-1, is a catheter containing stem cells injects these cells into the heart, with the goal of differentiating into heart cells, and developing new tissue, is the probable method that will be used in cellular cardiomyoplasty.

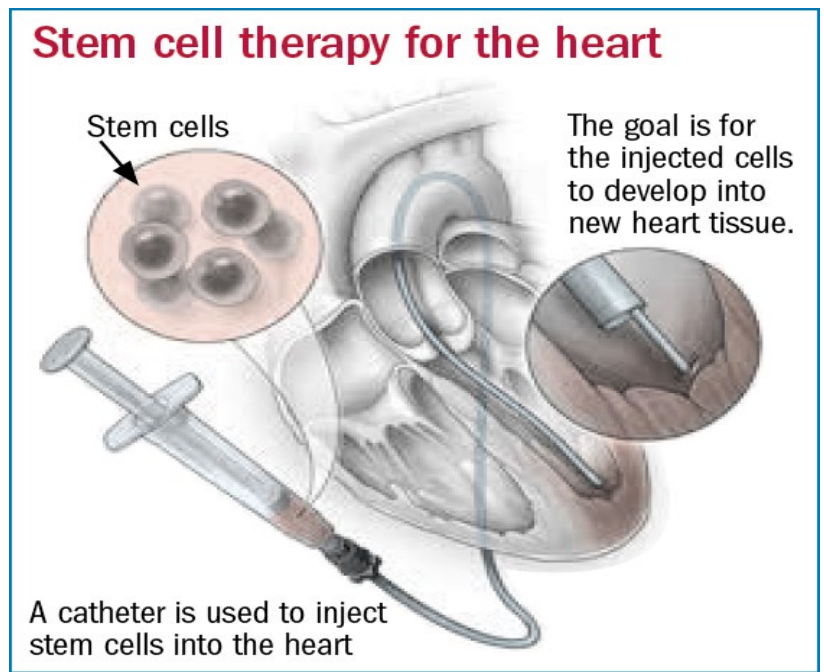

Figure 1. Stem cell therapy for the heart. Figure 1 shows a possible process for cellular cardiomyoplasty.

\section{Mesenchymal Stromal Cells}

Mesenchymal stromal cells are larger stromal cells (mesenchymal stem cells) that have the ability to exhibit multilineage differentiation of mesodermal, ectodermal, and endoderm such as muscle, neuron, liver cells, etc. [22, 34, 40]. These cells have been seen in mostly all of the tissues in the body, including umbilical cord, endometrial polyps, menses blood, bone marrow, adipose tissue, menstrual blood, and endometrium, as shown in Figure-2 [22]. Mesenchymal stromal cells and mesenchymal stem cells are different because mesenchymal stem cells is another way to say stromal cells, not mesenchymal stromal cells [40].

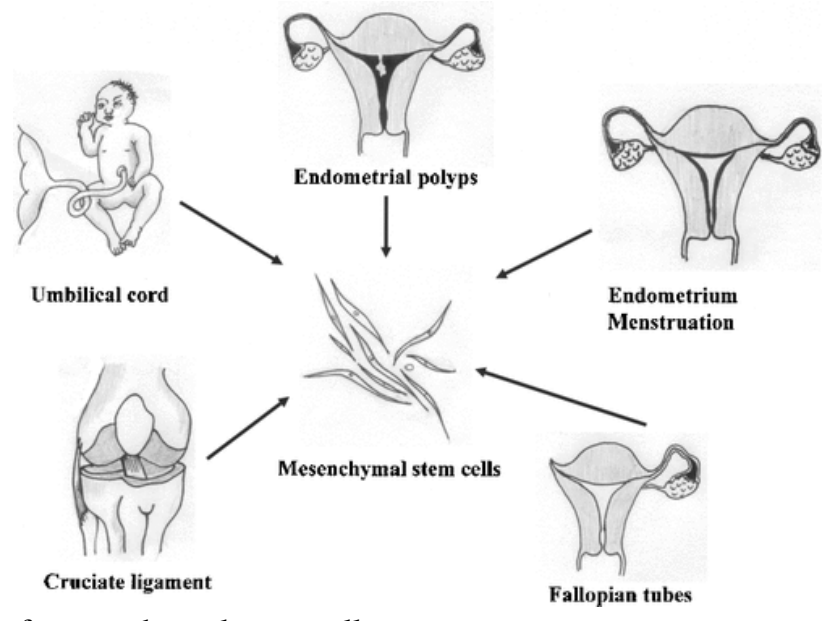

Figure 2. Primary locations of mesenchymal stem cells. 


\section{Function}

In vitro, or outside of the organism being used, mesenchymal cells have shown the ability of proliferation and multipotency. Because of this, these cells are being looked into as a possible treatment for heart failure and myocardial infarction, but also other degenerative and/or inflammatory diseases, as well as graft-versus-host diseases [25]. However, because there is not a lot known about these cells, whether they can cause long term issues, or what is the form that they work best in, scientists are still conducting tests to figure out what is the best way to obtain them, what is their genetic stability, their ability to repair tissue, etc.

Embryos and neonatal organisms (specifically referring to humans) do not have all of their cells like adults do. This is why different stem cells in the body are used to differentiate into specific cells with specific functions. Mesenchymal stromal cells have the ability to differentiate into a variety of cells including bone cells, cartilage, muscle cells, neural cells, skin cells, corneal cells, etc., as shown in Figure-3 [26]. Though they are found in many places in the body, they are primarily found in the bone-marrow and adipose tissue, and remain dormant until needed to promote healing in the body [25, 26, 27, 34]. As the body ages, mesenchymal cells age as well, slowly decreasing its effectiveness over the years [26].

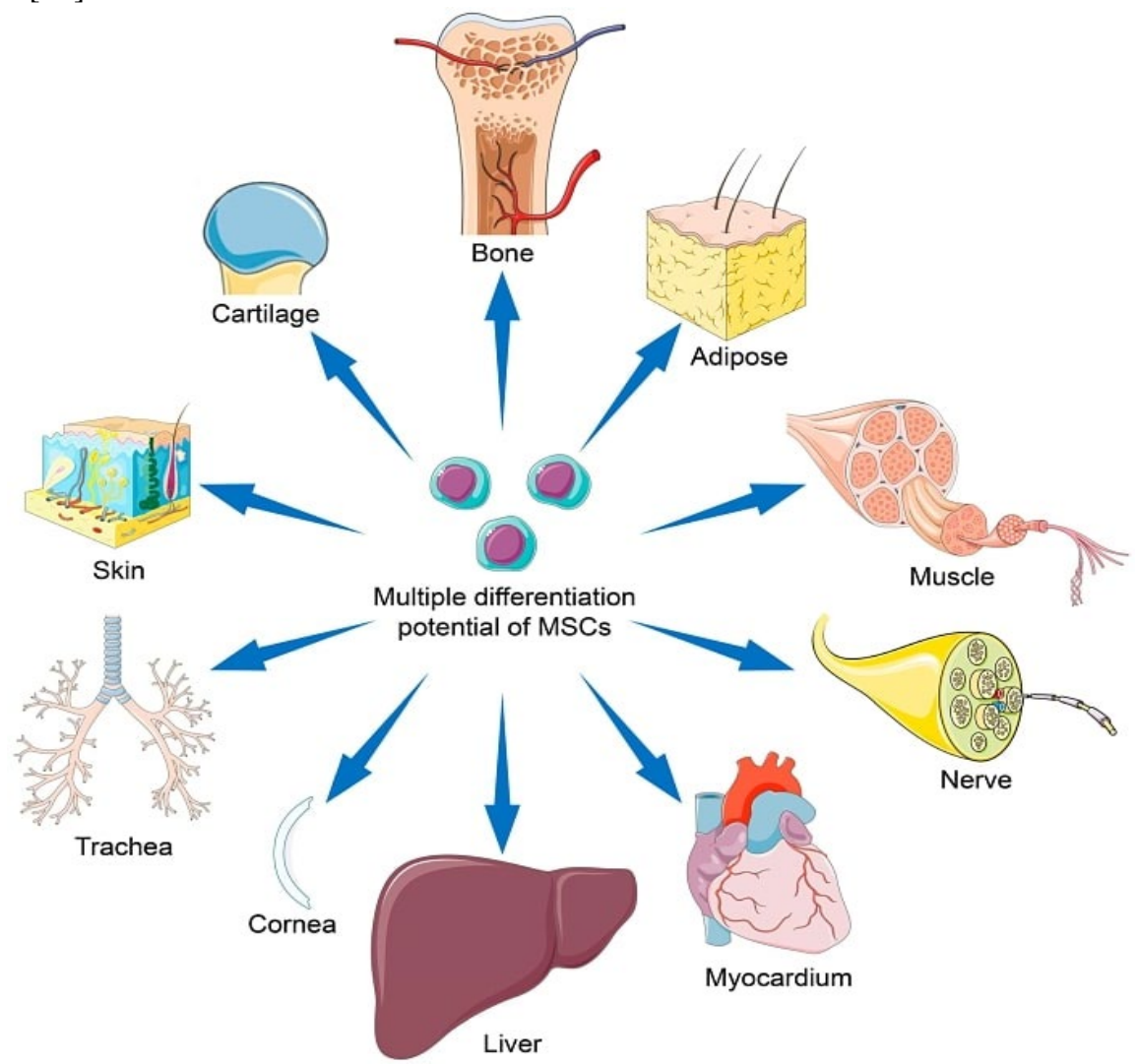

Figure 3. Multiple differentiation potential of MSCs. Shows different types of cells mesenchymal stromal cells can differentiate into.

\section{Mesenchymal Stromal Cells Properties}

\section{Immunomodulatory Properties}

Immunomodulatory properties are the ability of something to regulate the response of the immune system to a certain occurrence. When an acute myocardial infarction occurs, there is a pro-inflammatory response to it, with a purpose of removing debris and necrotic cells from the area of the heart where the blockage is occurring [27, 28]. This pro- 
inflammatory response does contribute, however to the death of cardiomyocytes, and manifests 6-24 hours after the infarction $[28,38]$. The immune system is specifically harmful in the heart during a myocardial infarction when its effects on the heart are prolonged for a certain period of time [36]. These $\mathrm{T}$ cells, which are a part of the immune system, specifically hurt the heart when they stimulate the formation of fibroids, which is explained more in detail in the next section. This then leads to the formation of scar tissue, causing heart failure.

In order to lessen the negative effects of the pro-inflammatory response in a myocardial infarction, mesenchymal stromal cells can regulate it by suppressing these white blood cells, and secreting anti-inflammatory subsets in innate immunity and adaptive immunity instead [27, 40]. With this property, mesenchymal stromal cells could be injected into the myocardium after a certain period of time of a heart attack, allowing the immune system to clear necrotic cardiomyocytes, but then coming in to regulate and prevent any adverse after-effects of it in the heart.

\section{Antifibrotic Effect}

The reason that myocardial infarctions lead to heart failure is because after a heart attack, excess collagen is deposited, leading to stiffness and the formation of scar tissue in the heart [27]. Because of the scar tissue, the heart is unable to contract at a normal and necessary efficiency, causing the heart to lose the ability to pump enough blood to other parts of the body. This pathological feature is specifically called myocardial fibrosis, because the necrotic cardiomyocytes were replaced with fibroblast [27]. Because contractile abilities are a specific characteristic to muscle cells in the heart, this fibroblast, not containing this skill, ultimately form a tissue that is too hard to help the function of the heart. Fibroblast hindering the ability for the heart to contract can be caused by $\mathrm{T}$ cells that, as stated above, can cause these fibroids to become harmful with time [36, 38].

Mesenchymal stromal cells (MSCs) have been seen to exhibit the antifibrotic effect. This means that when MSC's are injected into the myocardium, they can one: differentiate into cardiomyocytes with the ability to contract and cause the pumping of blood throughout the body, and two: stop the formation and replace the fibroblast with these new, and healthy cells.

In a 2014 study, scientists injected mesenchymal stromal cells into a three-dimensional in vitro co-culture model of cardiac fibrosis to evaluate the paracrine effects (the ability of a cell to produce a signal that alters the behaviour of other cells around it). After 48 hours of observing the effect of the cells on the fibroblast phenotype, these scientists concluded that mesenchymal stromal cells do lower the effect of the myofibroblast transition after a heart attack. [29]

\section{Properties: Mesenchymal Stromal Cells}

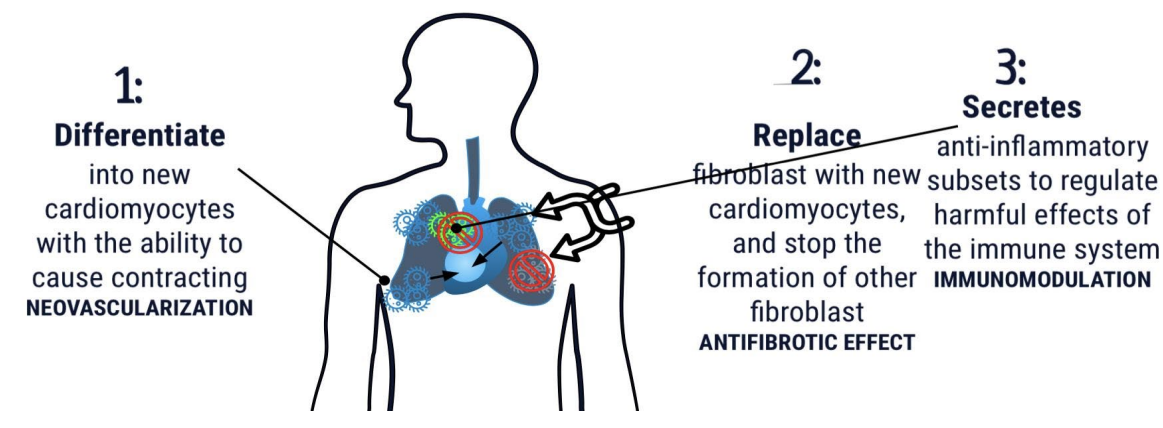

Figure 4. Properties of mesenchymal stromal cells. Seen in Figure 4, the three properties of mesenchymal stromal cells are neovascularization, the antifibrotic effect, and immunomodulation. 


\section{Cellular Cardiomyoplasty}

Cellular cardiomyoplasty is a process in which cells or other biological substances are grafted into the myocardium, to limit the consequences of diseases that cause the heart's contractile ability to weaken, or that damage the left ventricle [17]. Cellular cardiomyoplasty is considered to be a part of the cardiac regenerative medicine field, which has conducted experiments with the goal of it (cellular cardiomyoplasty) becoming an alternate approach to heart transplantation to treat patients especially with end-stage heart failure [18]. Cardiomyoplasty uses tissue engineering to stimulate the proliferation of myocardial cells. The goal of these cells is to become one with the native myocardium of an infarct heart, improving the contractile ability of the heart, hindering the ability or reversing the effects of scar tissue formation in the heart [34]. Biological substances or stem cells from other parts of the body are engrafted into the myocardium, in order for proliferation to occur. Bone marrow-derived cells to mesenchymal stromal cells to cellulose have been used to study cell therapy and determine its beneficial roles $[3,30]$.

\section{Deliverance Methods}

The figure 1 shows how the deliverance of cells in cellular cardiomyoplasty occurs, called minimally-invasive catheter delivery. A catheter is made from medical grade materials that form a thin tube [20, 21]. As Figure-1 demonstrates, these medical devices can be used to treat diseases or perform a surgical procedure, however their ability to provide a technique that can help treat diseases is what will be focused on here. They are specifically able to implant biomaterials that could permit a minimally invasive delivery of cell transplantation constructs and promote the formation of both new tissue and structures in the desired shape or sizes [21].

Intracoronary infusion is another delivery method for cellular cardiomyoplasty. This technique delivers stem cells through the coronary artery, usually through intracoronary catheterization [34]. The stem cells are infused using a catheter with a balloon attached to it, instead of the more syringe looking device in figure 1 . This method provides a maximum number of cells to target the intended area, with good blood supply containing oxygen crucial for cell survival [34]. As stated briefly before, mesenchymal stromal cells are larger in size, which cause damage to myocardium if there is microvascular obstruction. This is why intracoronary infusion may not be the most ideal form of transplantation and why finding the best delivery method for these cells is important, to ensure it is safe for the patient, and does not cause iatrogenic issues [34].

Another way to deliver molecules in cellular cardiomyoplasty is called intravenous peripheral infusion, or intravenous stem cells administration [34]. This easy deliverance method occurs with the homing, or migration of cells from their organ of origin, to the injury site in the heart [34]. Unfortunately, problems can occur with intravenous peripheral infusion, including a low amount of cells being delivered because only $3 \%$ of the normal cardiac output flows per minute through the left ventricle, and venous blood from the lungs may trap cells, causing a reduction of stem cells actually reaching the intended site[34].

The intramyocardial technique directly accesses the injured area usually with a needle tipped delivery catheter in the myocardium, allowing it to bypass the need for homing, mobilization, or risk the cells getting trapped in areas like the lungs [34]. However, because of the need to inject these cells right into the heart with this method, it can cause a hole to be created in the ventricle, or perforation [34], which may lead it to not be the best form of delivery.

\section{Clinical Studies}

Clinical studies are critical for ensuring the safety and efficiency of cellular cardiomyoplasty with mesenchymal stromal/stem cells. Preclinical studies have been used similarly for vaccines like the poliovirus vaccine in the 1900's, and the coronavirus vaccine, that is still being tested in different phases, in this kind of setting. Preclinical studies in particular help scientists to test certain aspects with models and other animals to ensure safety before moving on to 
testing humans in clinical studies. These preclinical studies and trials may test specific concepts like how MSC's work in the heart naturally after infarction, different modifications to the cells that may improve their function in different areas, etc. Additionally, when using animals, a left anterior descending artery ligation to replicate a bodily environment of a myocardial infarction is more commonly used than a technique to replicate the bodily environment of heart failure. Many of these clinical trials below will also be focused on the effects of mesenchymal stem cells regarding a myocardial infarction, to take a broader scope of these stromal cells (mesenchymal stromal cells).

\section{Cellular Cardiomyoplasty into Infarcted Swine's Hearts by Retrograde Infusion Through the Ve- nous Coronary Sinus}

This experimental study used two groups of fifteen (study group) and 10 (control group) juvenile farm pigs to create a model of myocardial infarction that would allow them to evaluate the retrograde infusion method for cellular cardiomyoplasty. The farm pigs underwent distal left anterior descending artery ligation for a month; after, the study group underwent sternotomy and were injected with a murine myoblastic line $\mathrm{C} 2-\mathrm{C} 12$ at a constant pressure of $30 \mathrm{mmHg}$, into the coronary sinus. After another 30 days, the living animals from both groups went through a trans echocardiography and 99Tc scintigraphy. The animals were later euthanized, and specimens were taken for microscopic evaluation. The results showed that after the ligation, there was a decrease in the cardiac output, and an increase for the animals that underwent cardiomyoplasty. Animals in the study group also showed a significant reduction of perfusion deficit in the left anterior descending artery territory, compared to those in the control group. It was concluded that venous retrograde infusion is safe, effective, and provides major improvement in the function and viability of the heart [19].

\section{MicroRNA-133a Engineered Mesenchymal Stem Cells Augment Cardiac Function and Survival Rate in the Infarct}

When MSC's are transplanted into an infarcted heart, it has been observed that the lack of oxygen in the ischemic environments causes apoptosis of a majority of the cells [23, 40], usually 48 hours after engraftment [40]. In 2015, scientists conducted a clinical trial in order to test whether engineering the genetics of mesenchymal stem cells could improve their survival rate during transplantation. These scientists tested specifically whether the overexpression of miR-133a in MSC's improves its survival and engraftment in the ischemic microenvironment of the heart. Isolated and plated MSC's were transplanted into Fisher-344 rats divided into four of five groups: one group was untreated, the second with normal treatment for myocardial infarction, the third treated with mesenchymal stem cells, the fourth with MSC's transfected with miR-133a mimic, and MSC's transfected with miR-133a antagomir. An in vivo model was used, where the left anterior descending coronary artery was under temporary ligation, and the Fisher-344 rats were anesthetized with $50 \mathrm{mg}$ of ketamine and $5 \mathrm{mg}$ of xylazine. The results showed that the hearts that were transplanted with miR-133a mimic led to a significant decrease in the infarct size, and increased cell engraftment [23].

\section{Differentiation of the Umbilical Cord Wharton's Jelly Derived Mesenchymal Stem Cells into Cardiomyocytes Using 5-Azacytidine}

As stated, before mesenchymal stem cells can be isolated in a variety of places including bone-marrow and adipose tissue. One area they can also be found is in umbilical cord blood. One study that shows this was conducted around 2017-2018. Scientists used blood samples from pregnant women due to deliver between the dates of October 2015 and April 2017. Mesenchymal stem cells were successfully cultured and isolated from umbilical cord tissue (which is the Wharton's jelly). Additional information obtained from the results of this study is that mesenchymal stem cells can differentiate into cardiomyocytes when subcultured in differentiating media containing azacytidine [32]. 


\section{Mesenchymal Stem Cells from Human Amniotic Membrane Differentiate into Cardiomyocytes and Endothelial-Like Cells Without Improving Cardiac Function After Surgical Administration in Rat Model of Chronic Heart Failure}

When looking for new possible treatments for large-scale affecting diseases like heart failure, objectivity is necessary in every step. Though the use of mesenchymal stem cells in cellular cardiomyoplasty to treat certain cardiomyopathies has shown potential of use in a variety of studies, there have been studies that did not show positive results. In this study, scientists tested the efficiency of human amino-derived mesenchymal stem cells on the treatment of chronic model myocardial ischemia and heart failure in Male Wistar rats. The rats were divided into three groups: sham, the control, which only underwent left anterior descending artery ligation with no treatment, and the animals which were injected with the human amino-derived mesenchymal stem cells four weeks after undergoing left anterior descending artery ligation. The results showed that the injection of these mesenchymal stem cells did cause proliferation of cardiomyocytes, however, it did not cause improvement in cardiac function. The scientist concluded that human aminoderived mesenchymal stem cells are effective in the early phases of myocardial ischemia but do not offer any significant advantage in patients with chronic heart failure [33].

\section{Characterization of the Immunomodulatory Properties of Alveolar Bone Derived Mesenchymal Stem Cells}

Mesenchymal stem cells harbor immunomodulatory properties, as stated before, that allows them to treat inflammatory or inflammatory-causing conditions. Scientists conducted a study to observe these properties of mesenchymal stem cells, specifically alveolar bone derived mesenchymal stem cells and bone marrow-derived mesenchymal stem cells. Both kinds of cells were cultured in the same conditions, and were co cultured with different types of immune cells such as THP-1 monocytes, macrophages, and peripheral blood mononuclear cells to evaluate their effects on the proliferation, differentiation, and activation of the immune cells. Both alveolar bone-derived and bone marrow-derived mesenchymal stem cells drove macrophages into an anti-inflammatory phenotype, the alveolar bone-derived mesenchymal stem cells showing greater use of immunomodulatory properties than the bone marrow-derived mesenchymal stem cells [39].

\section{Discussion}

Cellular cardiomyoplasty has been gaining interest in the cardiac regenerative medicine field, using methods like intracoronary infusion, and intravenous peripheral infusion to deliver biomolecules into the heart, with the goal of causing the regeneration of dead or disabled myocardium. Substances that may be injected into the heart using the deliverance methods stated are usually stem cells which include but are not limited to bone marrow-derived stem cells, embryonic stem cells, and mesenchymal stem cells. Deliverance methods that are currently being looked into include retrograde infusion, intracoronary infusion, intravenous peripheral infusion, and the intramyocardial technique. There have been obvious problems with these techniques that have been found, and as research grows, a new deliverance technique should continue to be looked for in order to ensure the most ideal and effective way to deliver cells with cellular cardiomyoplasty.

Mesenchymal stromal cells, the specific term for mesenchymal stem cells usually accompanied with the histological origin of the cells are used by the body naturally to promote healing, and remain dormant until needed. These cells have been seen in almost all of the body's tissues, however they can primarily be found in the bone marrow and adipose tissue. The issue with obtaining MSCs from the bone marrow is that it involves an invasive procedure, so the fact that they are found in various places may be useful, in case there is a number of tissues where it is unethical to retrieve them for therapy, as research and studies on them continue to grow. 
Mesenchymal stromal cells have been seen to all harbor two important qualities which make it possible to be used in cellular cardiomyoplasty to treat cardiomyopathies, specifically heart failure and the harmful effects of a myocardial infarction: immunomodulatory properties, and antifibrotic effect. The immunomodulatory properties of mesenchymal stromal cells is the ability of the cells to suppress the effects of the immune system, usually after a myocardial infarction. This ability of MSCs allow them to stop the inflammation in the heart, which occurs in order to clear dead cardiomyocytes, which can later cause the formation of scar tissue, leading to chronic heart failure. Because the inflammatory response of the immune system after a heart attack has its benefits, cellular cardiomyoplasty most likely would be most useful with mesenchymal stromal cells a certain time span after the incident, instead of being immediately injected.

Hard textured tissue in the heart, or scar tissue, prohibits the heart from contracting in order to pump blood throughout the body. This is caused by the formation of fibroblast in the heart, which are used to replace the dead cardiomyocytes. Unlike the cells they replace, they do not have the ability to contract, which stops the heart from being able to act like a pump. The ability of mesenchymal stromal cells to exhibit the antifibrotic effect after being injected into the heart helps to prohibit the formation of scar tissue, and instead replace what would have been fibroblast with new cells in the heart, originally having been mesenchymal stromal cells.

\section{Conclusion and Implications}

Heart failure and myocardial infarction continue to be major causes of death and disability. The current treatments are unable to keep the numbers down because of their inefficiency, or the scarcity and lack of availability for them. Cellular cardiomyoplasty, with its various deliverance techniques have shown the ability to keep up with demands from heart failure and myocardial infarction patients, as well as, with substances like mesenchymal stromal cells, the ability to help reverse the effect of these two cardiomyopathies. Because of MSCs ability to regenerate new tissue in the heart, control the immune systems response to a myocardial infarction, and stop the formation of fibroblast that in time harm the heart, if they prove to be entirely safe in the highest levels of their testing, mesenchymal stromal cells administered through cellular cardiomyoplasty may be used as a treatment for heart failure and mesenchymal stromal cells in the future. If fully approved, MSC's can be used in the cardiac field, not only for the two stated diseases, but also for other cardiomyopathies that affect the heart tissue, affecting its ability to pump blood.

\section{Limitations and Future Research}

Cellular cardiomyoplasty still has a long way to go before it can become an official treatment for patients whose hearts are progressing or already are at the stage of heart failure. There are many biological substances being used in studies for cellular cardiomyoplasty including mesenchymal stromal cells. Though they have proved many times that they have a large potential of use for cardiomyoplasty, future studies should aim to define the optimal dose that will help patients with the above diseases to have smooth recoveries. This dose may vary between sex, age, race, weight, etc. and should be test to get a general dosage amount for a range in each category. Scientists should also test how long after a myocardial infarction mesenchymal stromal cell should be administered, since the initial response of T-cells are not harmful. Future research should also look into the best deliverance technique that will let the cells function at their best ability and if it varies between patients, without any iatrogenic consequences, since some deliverance techniques may pose danger to perforation. If mesenchymal stromal cells move up phases of studies to get nearer to being used therapeutically, scientists should also look for whether there are certain ways to personally help a specific patient, whether different modifications of the stromal cells can help one person better than the other [31]. They should also see if taking stromal cells from a patient and delivering it back into the patient to respond to heart failure and myocardial infarction produces better results than taking stromal cells from a source and administering it to everyone. 


\section{Data Analysis}

To gather research papers, the key words "cellular cardiomyoplasty", "myocardial infarction", "heart failure", "mesenchymal stromal cells", "immunomodulatory properties", and "antifibrotic effect" were input into Google Scholar, PubMed, ERIC, Med Ed Portal, and Google (all sources from google were verified as reliable sources). All hits were input into a Google Sheet and separated by search engine/database, and papers went through one stage of elimination of title relevancy, and another regarding abstract relevancy. Out of the 369 hits from all of the engines/databases, 39 were kept, 23 from Google Scholar, 12 from Pubmed, 0 from ERIC, 0 from Med Ed Portal, and 4 from Google. Then, the 39 research papers were fully read, and rated on relevancy. Of the 39 papers, 29 were kept, 22 from Google Scholar, 3 from PubMed, and 4 from Google. Papers were then categorized by article type, relation to this review paper, and strength of finding. 11 extra articles were found and were put through the same process, but were not input into the spreadsheet: "Origin of Cardiomyocytes in Adult Heart", "Tissue Engineering Strategies for Myocardial Regeneration: Acellular Versus Cellular Scaffolds?", "Therapeutic Approach for Regenerating the Myocardium", "Deaths from Heart Failure Vary by Geography", "Heart Failure", "Ethical Issues in Stem Cell Research", "FDA Warns About Stem Cell Therapies", "Cellular Cardiomyoplasty", "Shape-defining scaffolds for minimally invasive tissue engineering", "Mesenchymal Stem Cells", "Biological functions of mesenchymal stem cells and clinical implications".

\section{Acknowledgements}

I would like to thank Leya Jokutty for being my mentor and instructor through the process of writing this review paper.

\section{References}

1. Leri, A., Rota, M., Pasqualini, F. S., Goichberg, P., \& Anversa, P. (2015, January 2). Origin of Cardiomyocytes in Adult Heart. PubMed Center. Retrieved September 30, 2020, from https://www.ncbi.nlm.nih.gov/pmc/articles/PMC4283577/\#!po=0.847458

2. Domenech, M., Polo-Corrales, L., Ramirez-Vick, J. E., \& Freytes, D. O. (2016,

December 1). Tissue Engineering Strategies for Myocardial Regeneration:

Acellular Versus Cellular Scaffolds? PubMed Central. Retrieved September

30, 2020, from https://www.ncbi.nlm.nih.gov/pmc/articles/PMC5124749/\#s001title

3. Heallen, T. R., Kadow, Z. A., Kim, J. H., Wang, J., \& Martin, J. F. (2019, May

23). Stimulating Cardiogenesis as a Treatment for Heart Failure.

Circulation Research. Retrieved September 30, 2020, from

https://www.ahajournals.org/doi/full/10.1161/CIRCRESAHA.118.313573

4. Piano, M. R., \& Carrigan, T. M. (2003, December). Cellular Cardiomyoplasty A New

Therapeutic Approach for Regenerating the Myocardium. NursingCenter.

Retrieved September 30, 2020, from

https://www.nursingcenter.com/journalarticle?Article ID=447333\&Journal ID $=54006 \&$ Issue ID $=447308$. 
5. Pendyala, L., Gadesam, R., Ghiu, I., Hou, D., \& Chronos, N. (2008, June 19). Cellular Cardiomyoplasty and Cardiac Regeneration. CathLabDigest.

Retrieved October 1, 2020, from https:/www.cathlabdigest.com/articles/Cellular-Cardiomyoplasty-andCardiac-Regeneration

6. Deaths from Heart Failure Vary by Geography. (n.d.). Centers for Disease Control and Prevention. Retrieved October 1, 2020, from https://www.cdc.gov/heartdisease/heart failure.htm

7. Heart Failure. (n.d.). National Heart, Lung, and Blood Institute. Retrieved October 1, 2020, from https://www.nhlbi.nih.gov/health-topics/heart-failure

8. Heart Attack (Myocardial Infarction). (2019, February). Harvard Health

Publishing. Retrieved October 1, 2020, from https://www.health.harvard.edu/a to z/heart-attackmyocardial-infarction-a-to-z

9. Thygesen, K., Alpert, J. S., \& White, H. D. (2007, October 19). Universal

Definition of Myocardial Infarction. Circulation. Retrieved October 1, 2020, from https:/www.ahajournals.org/doi/full/10.1161/circulationaha.107.187397

10. Bayés-Genis, A. (2020, April 24). Heart Failure Treatment by Device. PubMed Central. Retrieved October 1, 2020, from https:/www.ncbi.nlm.nih.gov/pmc/articles/PMC7199188/

11. Medications Used to Treat Heart Failure. American Heart Association. Retrieved October 1, 2020, from https://www.heart.org/en/health-topics/heart-failure/treatment-options-for-heart-failure/medicationsused-to-treat-heart-failure

12. Mayo Clinic Staff. (n.d.). Heart Disease. Mayo Clinic. Retrieved October 1, 2020, from https://www.mayoclinic.org/diseases-conditions/heart-disease/symptoms-causes/syc-20353118

13. Lo, B., \& Parham, L. (2009, April 14). Ethical Issues in Stem Cell Research. Retrieved October 2, 2020, from https://www.ncbi.nlm.nih.gov/pmc/articles/PMC2726839/\# sec5title

14. FDA Warns About Stem Cell Therapies. (2019, September 3). FDA. Retrieved October 3, 2020, from https:/www.fda.gov/consumers/consumer-updates/fda-warns-about-stem-cell-therapies

15. Stem cells to repair heart damage? Not so fast. (2018, January). Harvard Health Publishing. Retrieved October 3, 2020, from https://www.health.harvard.edu/heart-health/stem-cells-torepair-heart-damage-not-so-fast

16. Heart Attack Symptoms, Risk, and Recovery. (n.d.). Center for Disease Control and Prevention. Retrieved October 7, 2020, from https://www.cdc.gov/heartdisease/heart attack.htm

17. Pendyala, L., Goodchild, T., Gadesam, R. R., Chem, J., Robinson, K., Chronos, N., \& Hou, D. (2008, May 4). Cellular Cardiomyoplasty and Cardiac Regeneration. PubMed Central. Retrieved October 7, 2020, from https://www.ncbi.nlm.nih.gov/pmc/articles/PMC2779354/ 
18. Dorin, R. P., \& Koh, C. J. (2011). Cellular Cardiomyoplasty. Science Direct. Retrieved October 7, 2020, from https://www.sciencedirect.com/topics/agricultural-and-biological-sciences/cellular-cardiomyoplasty

19. Prifti, E., Lascio, G., Harmelin, G., Bani, D., Briganti, V., Veshti, A., \& Bonacchi, M. (2016, February 13). Cellular cardiomyoplasty into infracted swine's hearts by retrograde infusion through the venous coronary sinus: An experimental study. Retrieved November 06, 2020, from https://www.sciencedirect.com/science/article/pii/S1553838916300185

20. Steele, A., Paulsen, M., Wang, H., Stapleton, L., Lucian, H., Eskandari, A., Hironaka, C. E., Farry, J. M., Baker, S. W., Thakore, A. D., Jaatinen, K. J., Tada, Y., Hollander, M. J., Williams, K. M., Seymour, A. J., Totherow, K. P., Yu, A. C., Cochran, J. R., Woo, Y. (2020, January 21). Multi-phase catheter-injectable hydrogel enables dual-stage protein-engineered cytokine release to mitigate adverse left ventricular remodeling following myocardial infarction in a small animal model and a large animal model. Retrieved November 06, 2020, from https://www.sciencedirect.com/science/article/abs/pii/S104346661930403X

21. Thornton, A. J., Alsberg, E., Albertelli, M., \& Moone, D. J. (2004). Shape-defining scaffolds for minimally invasive tissue engineering. Microsoft Academic. Retrieved November 06, 2020, from https://academic.microsoft.com/search?q=shape+defining+scaffolds+for+minimally+invasive+tissue+engineeri $\underline{\text { ng}}+$ catheter + delivery + procedure

22. Dah-Ching Ding, W. (2011, February 1). Mesenchymal Stem Cells - Dah-Ching Ding, Woei-Cherng Shyu, Shinn-Zong Lin, 2011. PubMed. Retrieved November 06, 2020, from https://journals.sagepub.com/doi/full/10.3727/096368910X

23. Division of Pulmonary and Critical Care Medicine, Dakhlallah, D., Zhang, J., Yu, L., Marsh, C., Angelos, M. G., \& MPharm, M. (n.d.). MicroRNA-133a Engineered Mesenchymal Stem Cells Augment... : Journal of Cardiovascular Pharmacology. PubMed. Retrieved November 06, 2020, from https://pubmed.ncbi.nlm.nih.gov/25658461/

24. Gathier, W., Van Ginkel, D., Van der Naald, M., Van Slochteren, F., Doevendans, P., \& Chamuleau, S. (2018, February 1). Retrograde Coronary Venous Infusion as a Delivery Strategy in Regenerative Cardiac Therapy: An Overview of Preclinical and Clinical Data. PubMed. Retrieved November 06, 2020, from https://www.ncbi.nlm.nih.gov/pubmed/29392536

25. Naji, A., Eitoku, M., Favier, B. et al. (2019) Biological functions of mesenchymal stem cells and clinical implications. Springer Link. https://doi.org/10.1007/s00018-019-03125-1

26. Louis A. Cona, M. (2020, July 17). What are Mesenchymal Stem Cells (MSCs)? Retrieved November 06, 2020, from https://www.dvcstem.com/post/what-are-mesenchymal-stem-cells

27. Guo, Y., Yu, Y., Hu, S., Chen, Y., \& Shen, Z. (2020, May 11). The therapeutic potential of mesenchymal stem cells for cardiovascular diseases. Retrieved November 06, 2020, from https://www.nature.com/articles/s41419$\underline{020-2542-9}$ 
28. Ong, S., Hernández-Reséndiz, S., Crespo-Avilan, G., Mukhametshina, R., Kwek, X., Cabrera-Fuentes, H., \& Hausenloy, D. (2018, June). Inflammation following acute myocardial infarction: Multiple players, dynamic roles, and novel therapeutic opportunities. PubMed. Retrieved November 06, 2020, from https://www.ncbi.nlm.nih.gov/pmc/articles/PMC5981007/

29. Galie, P. A., \& Stegemann, J. P. (2014, July 16). Injection of mesenchymal stromal cells into a mechanically stimulated in vitro model of cardiac fibrosis has paracrine effects on resident fibroblasts. PubMed. Retrieved November 06, 2020, from https://pubmed.ncbi.nlm.nih.gov/24713331/

30. Simeoni, R., Francisco, J. C., Miyague, N., Irioda, A. C., Souza, C. M., Souza, D., et al. (2020, October 28). Beneficial Roles of Cellulose Patch-Mediated Cell Therapy In Myocardial Infarction: A Preclinical Study. Retrieved November 06, 2020, from https:/www.preprints.org/manuscript/202010.0573/v1

31. EJ. Benjamin, M., P. Ponikowski, A., D. Orlic, J., LH. Lund, L., MG. St John Sutton, N., Jugdutt, B., et al. (2020, August 12). Stem Cell Therapy for Chronic and Advanced Heart Failure. Springer Link. Retrieved November 06, 2020, from https://link.springer.com/article/10.1007/s11897-020-00477-9

32. Fathy WM, NourEldin RI, Shalby GH. (2019). Differentiation of umbilical cord Wharton's jelly derived mesenchymal stem cells into cardiomyocytes using 5-azacytidine. Menoufia Med J http://mmj.eg.net/article.asp?issn=11102098; year $=2019 ;$ volume $=32 ;$ issue $=3 ;$ spage $=991$; epage $=995 ;$ aulast $=$ Fathy

33. Gorjipour, F., Hosseini-Gohari, L., Alizadeh Ghavidel, A., Hajimiresmaiel, S., Naderi, N., Darbandi Azar, A., \& Pazoki-Toroudi, H. (2019, February 25). Mesenchymal stem cells from human amniotic membrane differentiate into cardiomyocytes and endothelial-like cells without improving cardiac function after surgical administration in rat model of chronic heart failure. PubMed. Retrieved November 08, 2020, from https://www.ncbi.nlm.nih.gov/pmc/articles/PMC6477105/

34. Nursalim, A., Katili, P., \& Santoso, T. (2016). Cellular Cardiomyoplasty For Myocardial Infarction: A 2014 Evidence-based Update. Acta Medica Indonesia. Retrieved November 08, 2020, from http://actamedindones.org/index.php/ijim/article/view/83

35. Gabisonia, K., Prosdocimo, G., Aquaro, G.D. et al. (2019) MicroRNA therapy stimulates uncontrolled cardiac repair after myocardial infarction in pigs. Nature 569, 418-422 https://doi.org/10.1038/s41586-019-1191-6

36. Rieckmann, M., Delgobo, M., Gaal, C., Büchner, L., Steinau, P., Reshef, D., et al. (2019, August 13). Myocardial infarction triggers cardioprotective antigen-specific T helper cell responses. J Clin Invest. 2019;129(11):4922-4936. Retrieved November 09, 2020, from https://www.jci.org/articles/view/123859

37. Chapman, A., Shah, A., Lee, K., Anand, A., Francis, O., Adamson, P., et al. (2017, November 17). Long-Term Outcomes in Patients With Type 2 Myocardial Infarction and Myocardial Injury. Circulation, 137(12),12361245. Retrieved November 09, 2020, from https://www.ahajournals.org/doi/full/10.1161/circulationaha.117.031806

38. Bacmeister, L., Schwarzl, M., Warnke, S. et al. (2019). Inflammation and fibrosis in murine models of heart failure. Basic Res Cardiol 114(19) https://doi.org/10.1007/s00395-019-0722-5 
39. C. Loebel, J., Mahla, R., A. Polymeri, W., N. D'Souza, F., TS. Windt, L., A. Singh, A., et al. (2020, March 05). Characterization of the immunomodulatory properties of alveolar bone-derived mesenchymal stem cells. Stem Cell Research \& Therapy, 11,102. Retrieved November 10, 2020, from https://link.springer.com/article/10.1186/s13287-020-01605-X

40. Caplan, A., AJ. Friedenstein, J., AJ. Friedenstein, S., AJ. Friedenstein, R., AJ. Friedenstein, R., AJ. Friedenstein, R., et al. (2020, August 08). Mesenchymal stromal cell therapies: Immunomodulatory properties and clinical progress. Stem Cell Research and Therapy, 11(345) Retrieved November 10, 2020, from https://stemcellres.biomedcentral.com/articles/10.1186/s13287-020-01855-9 